\title{
CrMYC1 transcription factor overexpression promotes the production of low abundance terpenoid indole alkaloids in Catharanthus roseus
}

\author{
Sima Sazegari ${ }^{1}$, Ali niazi ${ }^{2}$, Farajollah Shahriari-Ahmadi ${ }^{1}$, Nasrin Moshtaghi ${ }^{1}$, Younes Ghasemi ${ }^{3}$ \\ ${ }^{1}$ Department of Plant Breeding and Biotechnology, Ferdowsi University of Mashhad, Iran \\ ${ }^{2}$ Institute of Biotechnology, Collage of Agriculture, Shiraz University, Iran \\ ${ }^{3}$ Department of Pharmaceutical Biotechnology, School of Pharmacy, Shiraz University of Medical Sciences, Iran
}

\section{*Corresponding auther: shahriari@um.ac.ir}

\section{Abstract}

Catharanthus roseus is known as the only source for the low-abundance anticancer agents namely vinblastine and vincristine. Fine tuning of accumulation of such secondary metabolites is highly governed by the regulatory genes. Among these genes, Catharanthus roseus MYC1 (CrMYC1) is known as one of the key transcription factors regulating the biosynthesis of terpenoid indole alkaloid metabolites in C. roseus. In this study, CrMYC1 coding sequence (AF283506) was isolated and cloned in PBI121 plant binary vector. Then, CrMYC1 was transiently overexpressed in C. roseus leaves using agroinfiltration method. In addition to molecular analysis for confirming CrMYC1 overexpression, the profile of some chief terpenoid indole alkaloids in control and transgenic plants was evaluated by HPLC to elucidate the role of CrMYC1 in an increased in the anticancer components. The results indicated that overexpression of CrMYC1 transcription factor can increase most important terpenoid indole alkaloids including vinblastine, vincristine, and catharanthine in C. roseus. HPLC analysis of catharanthine and vinblastine contents showed about 3 and 2.5 fold increases, respectively, while the increase in vinecristine was not significant compared to that of the control. Therefore, CrMYC1 is introduced as an efficient candidate for manipulating TIA pathway in C. roseus and increasing at least the most valuable terpenoid indole alkaloids in this plant.

Keywords: catharanthine; metabolic engineering; regulatory gene; transient expression; vinblastine; vincristine.

Abbreviations: CrMYC1_Catharanthus roseus MYC1; TIA_terpenoidindole alkaloids; MEP_methyl D-erythritol 4-phosphate pathway; PRX1_peroxidase1; bHLH family_Helix loop Helix family; MeJ_methyl jasmonate; JA_Jasmonic acid; LB|_Luria-Bertani; OD_Optical density; npt II_Neomycin Phosphotransferase; STR_strictosidine synthase; TDC_tryptophan decarboxylase.

\section{Introduction}

Catharanthus roseus L. G. Don (Madagascar periwinkle) is an important dicotyledonous medicinal plant belonging to the Apocynaceae family. This plant is known as a source of producing more than 100 alkaloids some of which are very important natural pharmaceutical products. Among these alkaloids, Vinblastine, Vincristine, Ajmalicine, Serpentine, Vindoline, and Catharanthine are accounted for most important terpenoid indole alkaloids in this plant (Jacobs et al., 2004). Vinblastine and vincristine have been used as chemotherapeutics in the treatment of lymphoma and leukemia (Gidding et al., 1999). Ajmalicine and Serpentine are sometimes used as anti-hypertensive agents. In addition, it has been reported that major alkaloids such as vindoline have high antidiabetic activities particularly against type 2 diabetes (Nammi et al., 2003; Tiong et al., 2013). Generally, terpenoid indole alkaloids (TIA) are produced by combining the product of two independent pathways namely MEP and Shikmate that produce secologanin and tryptamine, respectively. This combination is made by a key enzyme known as STR, which generates strictosidine. Strictosidine as a major mediator embedded in the TIA pathway is converted to all different indole alkaloids such as ajmalicine, catharanthine, and vindoline in different branches. As catharanthine and vindoline are combined with PRX1 enzyme, they produce higher indole alkaloids vinblastine and vincristine in a multi-step biochemical reaction (Zhu et al., 2014).

Despite the significance of this natural metabolite in Catharanthus, the application of these bio-products is limited due to their very low yields. The total amount of vinblastine produced by $C$. roseus is $0.01 \%$ of its dry weight. Still, this volume is even lower in case of vincristine (0.0003\%) (Zhao and Verpoorte 2007). Numerous studies have been conducted for improving and increasing the amount of these significant natural metabolites in $C$. roseus (Zhao et al., 2013; Zhou et al., 2009). Biotechnology-based alkaloid enrichment programs provide a promising solution to overcome the obstacle of the TIA improvement in this plant (Mujib et al., 2012). Complexity in the TIA and its regulatory pathways that control TIA production can be considered as one of the limitations in the large-scale production of TIAs (Shanks 2005). In fact, TIAs in C. roseus are produced by a complex multi-step biosynthetic pathway known as TIA pathway. Moreover, their biosynthesis is 
highly regulated by developmental, cellular, and molecular stages (Dutta et al., 2007). TIA production pathway is highly regulated at transcription level by different transcription factors, some of which working as transcriptional activators and some as repressors (Verma et al., 2014). However, very limited information is available about any of these regulators (Menke et al., 1999; Suttipanta et al., 2011; van der Fits et al., 2000; Zhang et al., 2011).

Among all efforts made to enhance the major alkaloid content in $C$. roseus, manipulation of a regulatory gene can be named as one of the most promising strategies (El-Sayed and Verpoorte 2007). Regulating a group of genes, which may lead to an increase in the metabolites through employing transcription factors, is the advantage of this strategy in comparison with the manipulation of one enzymatic gene in TIA pathway (Broun et al., 2006). CrMYC1 is one of the putative TIA pathway regulators belongs to the bHLH transcription factors family. The effect of CrMYC1 on the regulation of terpenoid indole alkaloid genes, enzymes, and the metabolites content has not been studied yet. CrMYC1 expression is induced by methyl JA signaling pathway and fungal elicitor (El-Sayed and Verpoorte 2007; Memelink et al., 2001; Pauwels et al., 2009).

Investigating the effect of CRMYC1 on Chief TIA yield through overexpressing this gene can be useful for enriching the most important metabolites in the TIA pathway. Hence, this research was conducted to explore the possibility of increasing the most important TIAs in $C$. roseus through transiently overexpression the CRMYC1 transcription factor as the first step in a long-term program.

\section{Results}

\section{MYC1 expression in Catharanthus leaves using} agroinfiltration method

CrMYC1 gene was transiently transferred to $C$. roseus leaves using agroinfiltration method by narrow insulin syringe (Fig. 1). According to the result of confirmation the transfer and expression of CrMYC1 gene, this method was successful for expression of the gene in $C$. roseus, which has many limitations for regeneration after gene transfer.

\section{Molecular confirmation of transiently overexpressed leaves by PCR and real-time PCR}

As the Catharanthus plant has its own CrMYC1 gene, to ensure the T-DNA transfer containing both CrMYC1 and kanamycin resistant genes, nptll genes can be used and confirmed by the PCR. The PCR analysis of npt II genes confirmed the T-DNA transfer to leaves from CrMYC1+PBI121 constructs (Fig. 2). To compare the expression of CrMYC1 in transgenic leaves with the control leaves and to confirm the overexpression of this gene at mRNA level, a quantitative real-time PCR was performed. The quantitative expression patterns of both leaves 48 and 72 hours after agroinfiltration of leaves for CrMYC1 gene show an increase compared to those of the control leaves (Fig. 3). The result showed that the mRNA level of CrMYC1 transcript, compared to the control line, has been increased 4 fold in overexpressed leaves after 48 hours of injection. However, the difference between the expression levels of CrMYC1 at 72 hours and control was low (Fig. 3).

\section{Analysis of alkaloids concentration in overexpressed versus} control leaves

In order to determine the effect of CrMYC1 overexpression on the level of indole alkaloids in C. roseus, the profiles of four different TIA alkaloids (Ajmalicine, catharanthine, vinblastine, and vincristine) were measured by HPLC in both overexpressed and control lines (Fig. 4). These alkaloids were identified by comparing the retention time and the UV spectra with standards. To ensure the peak purity, we used the AUTOchrom 3000 software. The standard curve of alkaloids was generated through the implementation of different levels of each standard employed as a reference. Next, the content of each alkaloid was quantified by comparing it with the standard curves. The content of each metabolite in overexpressed leaves was quantified and compared with those of controls. The values of all analyzed alkaloids are presented in Table 2. Different amounts of catharanthine, vinblastine, and vincristine were detected in both 48 and 72 hours overexpressed leaves in comparison with the control plants. The results showed a significant increase in the amount of Vinblastine and catharanthine after transient expression while the increase in vincristine was not significant (Fig. 5).

\section{Discussion}

A few studies have been accomplished on transient expression of TIA constructive enzymatic and reporter genes in C. roseus. Transient overexpression of TDC and STR gene in intact Catharanthus leaves have been accomplished and confirmed by Stephano Di Fiore et al. (2004), who demonstrated the transient accumulation of recombinant enzymes in Catharanthus leaves. However, they did not study the effect of transiently overexpressed TDC and STR1 genes on TIA pathway intermediates or products. They concluded that agroinfiltration is a suitable tool to express the TIA genes in C. roseus. According to the result of this study, CrMYC1 was successfully overexpressed by using agroinfiltration method. Nevertheless, it should be considered that transient expression can be affected by other variabilities including physiological, growth, and developmental state of the plant itself. Thus, it is suggested to perform a transient expression on a large number of plants and in different growth stages whenever possible. In general, the transient expression system used in this study is introduced as a suitable testing tool for the further development of a stable metabolic engineering program by CrMYC1 or other transcription factors.

Consistent with the hypothesis of this study, transient overexpression of CrMYC1 leads to an increase in CrMYC1 transcript. However, according to the small increase in CrMYC1 transcript after 72 hours, the suitable time for transient expression is 48 hours after leaves injection. Nevertheless, it will be more useful to check for some other intermediate time points particularly for expression of enzymatic proteins. Finally, to check whether CrMYC1 overexpression is successful for producing more amounts of TIA alkaloids, we examined the alkaloid levels.

Based on the HPLC analysis, Ajmalicine was not detected in any of extract samples. This result is in accordance with the most findings of the localization of Ajmalicine. It is 
Table 1. Different Alkaloid contents of transient overexpressed and control lines in Catharanthus roseus leaves (mg/g dry wt.)

\begin{tabular}{lccc}
\hline & Catharanthine & Vinblastine & Vincristine \\
\hline Control & $0.9575 \pm 0.051$ & $0.7925 \pm 0.029$ & $0.220 \pm 0.014$ \\
48hours & $3.085 \pm 0.075$ & $2.1625 \pm 0.050$ & $0.350 \pm 0.021$ \\
72hours & $2.175 \pm 0.202$ & $1.3975 \pm 0.078$ & $0.3625 \pm 0.054$ \\
\hline
\end{tabular}

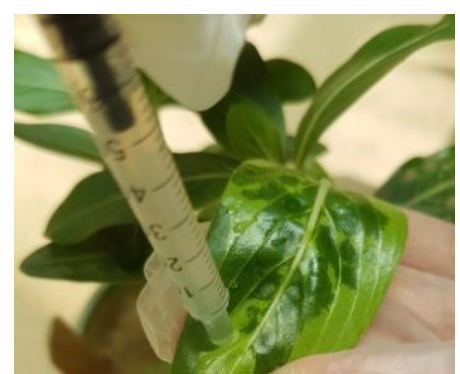

Fig 1. Agroinfiltration of Catharanthus leaves with Agrobacterium tumefaciens containing PBI121+CrMYC1 constructs.

Table 2. List of primers used in this study.

\begin{tabular}{|c|c|}
\hline Primer name & Primer sequence \\
\hline \multirow[t]{2}{*}{ CrMYC1 for isolation } & 5'CGGGATCCAACAGTTGATGGTGGTGGTG3' \\
\hline & 5'CGGAGCTCGCTTGGCTTCTCAATGCTCT3' \\
\hline \multirow[t]{2}{*}{ CrMYC1 for real-time analysis } & 5'AATCCTTACAGCGCCAGGT3' \\
\hline & 5'AAAACCACCACCAATCTGCA3' \\
\hline \multirow[t]{2}{*}{ Elongation factor $\alpha$} & 5'GCTTTACCTCCCAAGTCATCATC3' \\
\hline & 5'GGCTCCTTCTCAATCTCCTTACC3' \\
\hline \multirow[t]{2}{*}{ nptll } & 5'TTGGGTGGAGAGGCTATTCG 3' \\
\hline & 5'GAATCCAGAAAAGCGGCCAT3' \\
\hline
\end{tabular}

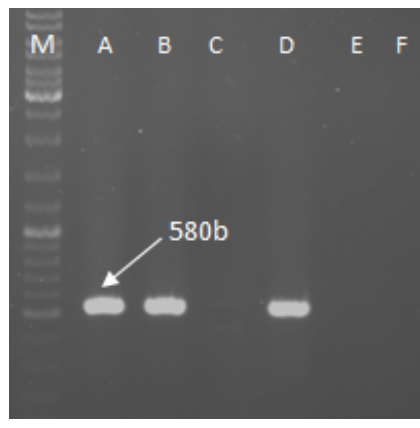

Fig 2. PCR analyses for the presence of the nptll gene. M) $100 \mathrm{bp}$ DNA marker, lane: A) $48 \mathrm{~h}$ after agroinfitration, B) $72 \mathrm{~h}$ after agroinfiltration, and C) Control line. D) Plasmid as positive control. E) Negative control in PCR.

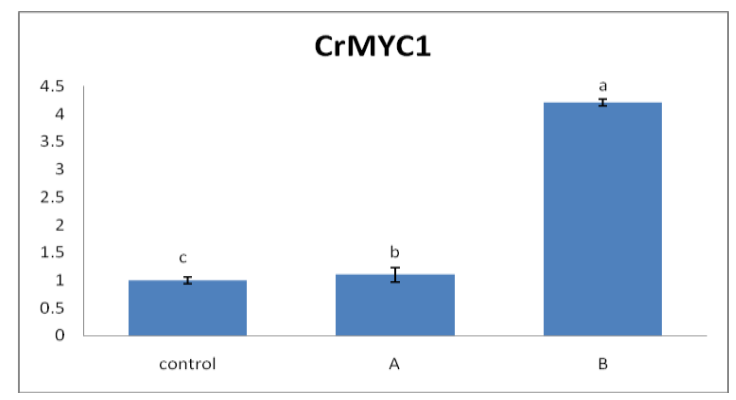

Fig 3. Real-time PCR for analysis CrMYC1 mRNA level in transiently overexpressed lines in comparison with control: A) $48 \mathrm{~h}$ after injection and B: $72 \mathrm{~h}$ after injection. Values are presented as the mean and standard deviation of triplicate samples. 

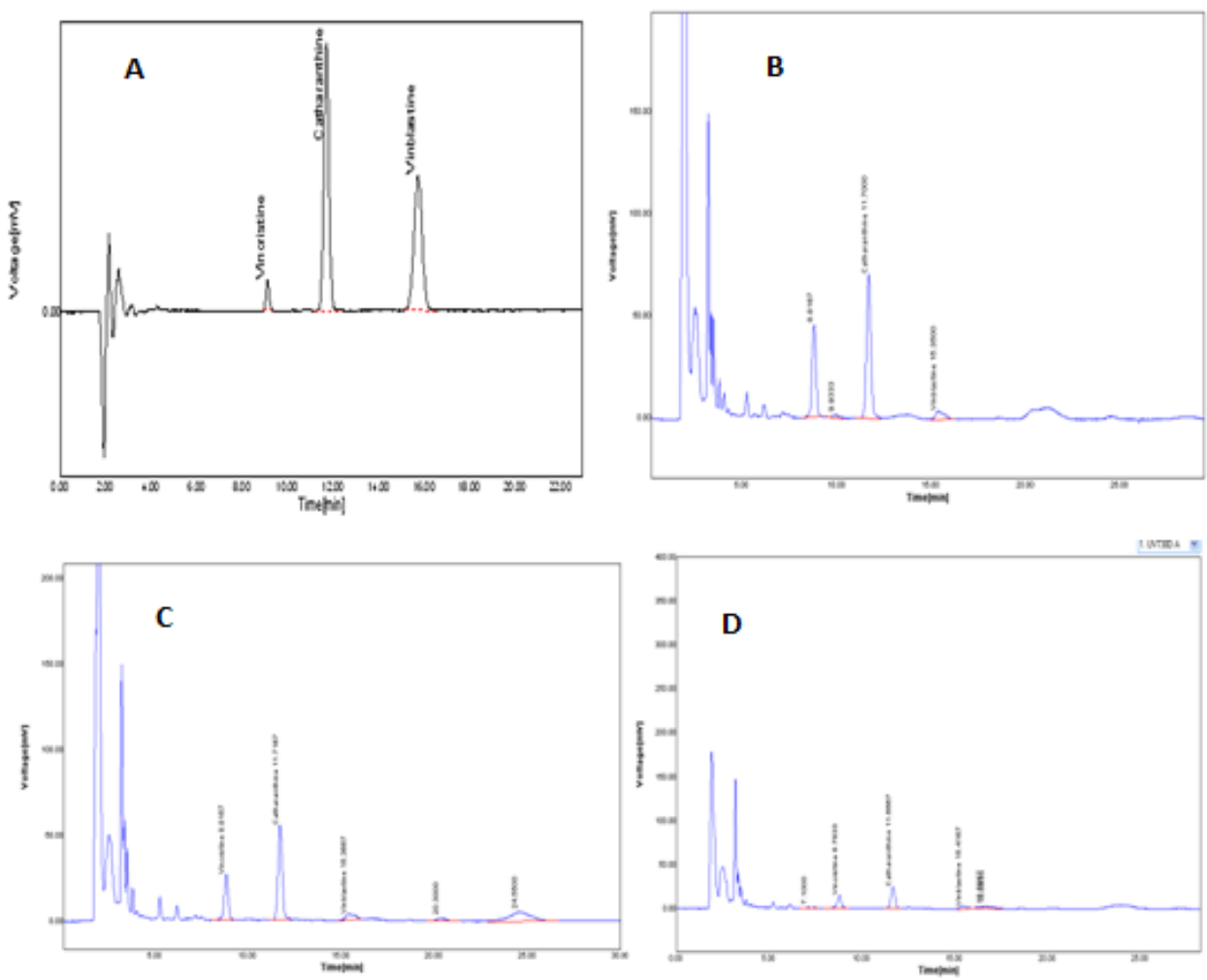

Fig 4. HPLC analyses of TIAs accumulation in transiently overexpressed leaves: A) Standard, B) $48 \mathrm{~h}$ after agroinfiltration, C) $72 \mathrm{~h}$ after agroinfiltration, and D) Control. The abscissas represent the retention time $(\mathrm{min})$ and the $y$-axes represent the absorbance.
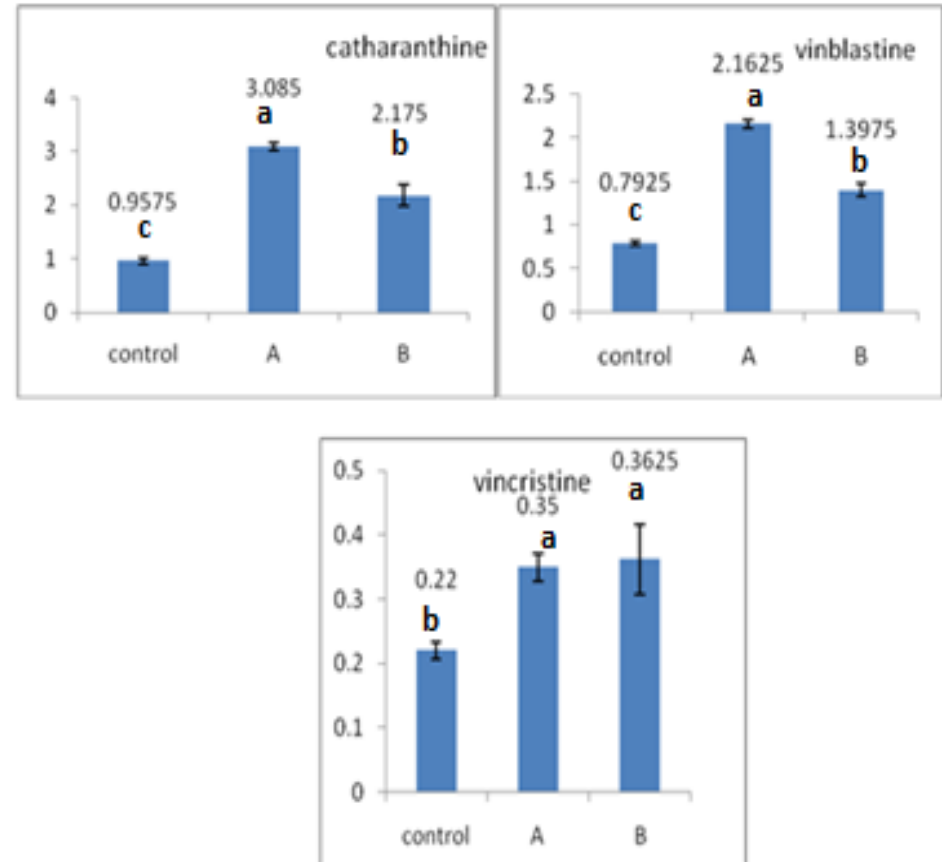

Fig 5. Catharanthine, vinblastine, and vincristine content in transiently overexpressed and control leaves: A) $48 \mathrm{~h}$ after agroinfiltration and B) $72 \mathrm{~h}$ after agroinfiltration. 
Catharanthus roots as the main source (El-Sayed and Verpoorte 2007). However, Hirata et al. (1978) reported Ajmalicine in the culture of leaves and the stem tissue of Catharanthus at a low level of $0.02 \mathrm{mg} / \mathrm{g}$ of dried weight. The accumulation of all three alkaloid shows an increase in CrMYC1 overexpressed lines (Table 1). Observing a significant increase in the amount of Vinblastine and catharanthine is probably due to the regulating mechanism of TIA pathway, which is governed by the CrMYC1 regulator directly or indirectly. It means that CrMYC1 is a potential transcription factor that induces expressions of some genes in the TIA pathway including STR and PRX1, which are responsible for catharanthine and vinblastine production. These findings confirm the result of Cathel et al. (2003) and Pre et al. (2000) who reported STR enzyme as a target for CrMYC1 transcription factor since they found that this transcription factor was bound to G-box element in the promoter of STR protein as bait in the two hybrid yeast system. So, it can be concluded that overexpression in CrMYC1 transcription factor causes an increase in the expression of some regulatory or enzymatic genes responsible for the catharanthine and vinblastine production. However, further chemical analyses on these enzymes can be also helpful to clarify if these enzymes or their functions increase through overexpression of $\mathrm{CrMYC1}$. Nevertheless, determining an increase in the yield or function of the enzymes in the TIA pathway through overexpression of CrMYC1 cannot confirm that they are the main targets of this transcription factor because CRMYC1 may indirectly enhance the function of some TIA enzymes by targeting another one or more regulatory genes. Similarly, overexpression of ORCA2 transcription factor in Catharanthus roseus hairy root resulted in a significant increase in catharanthine content but the amount of vinblastine and vincristine was below the detectable level ( $\mathrm{Li}$ et al., 2013). However, there are some limitations for detailed investigation since the catharanthine production pathway is not recognized genetically and enzymatically. Besides, the nonsignificant increase in vincristine might be stem from the fact that the part of the pathway that contains the conversion of vinblastine to vincristine is not governed by CrMYC1. Hence, a slight increase in vincristine is observed just due to the increase in vinblastine as a precursor. According to the two different time points after transient expression, the results indicated that the accumulation of catharanthine and vinblastine was at the highest level after 48 hours while vincristine was at the highest accumulation in 72 hours after transient expression (Fig. 5). Accumulation of catharanthine and vinblastine were 3.08 and $2.16 \mathrm{mg} / \mathrm{g}$ of dry weight, respectively, indicating a 3 -fold increase in transiently overexpressed lines compared to the control line. Interestingly, a close relationship between the highest mRNA level of CrMYC1 and highest alkaloid (catharanthine and vinblastine) content was seen within a period of 48 hours after leaves injection. In other words, the 4-fold-change in CrMYC1 transcript level in 48 hours after transient overexpression leads to the 3 -foldchange in vinblastine and catharanthine amount. This coincident result implied the role and distinction of CrMYC1 in improving the level of most important TIA in C. roseus.

\section{Materials and Methods}

\section{Plant materials}

C. roseus L. G. Don seeds were grown in the greenhouse under a controlled condition with natural daylight and $24 / 18^{\circ} \mathrm{C}$ day-night temperature. For gene isolation, onemonth plants were used after MeJ treatment with the 0.6 $\mathrm{mM}$ concentration. Besides, mature leaves of two-month plants were used for agroinfiltration.

\section{CrMYC1 isolation and cloning}

Total RNA was extracted from MeJ exposed leaves by Denazist RNA extraction kit after 24 hours and first strand CDNA was synthesized by CDNA synthesis kit (Amplisense Company). The reference gene for isolation was already submitted to NCBI database by accession number AF283506. CrMYC1 CDS was amplified and isolated by reverse transcription PCR (RT-PCR) using specific primers in Table 1. The 940 bp-isolated fragment was sequenced by Seqlab Company (Germany) and compared through using the Basic Local Alignment Search Tool (BLAST) service (http://www.ncbi.nlm.nih.gov/BLAST/) for the purpose of confirmation. The CrMYC1 isolated gene was cloned into PBI 121 plant binary vector containing the CaMV-35S promoter and NOS terminator to generate the CrMYC1 expression construct. BamHI and Sacl restriction enzymes were used for both CrMYC1 and PBI vector digestion and subsequently, ligation was done for directional cloning procedure. Agrobacterium tumefaciens strain 15834 was transformed with PBI121-CrMYC1 recombinant construct through the freeze-thaw method.

\section{Transient overexpression of CrMYC1 in Catharanthus}

A single transformed colony of recombinant Agrobacterium tumefaciens was cultured in the $20 \mathrm{ml}$ LB media containing $50 \mathrm{mg} / \mathrm{l}$ kanamycin and grown up at $28^{\circ} \mathrm{C}$ and $150 \mathrm{rpm}$ for $16 \mathrm{~h}$. The culture was centrifuged at $4000 \mathrm{rpm}$ for $15 \mathrm{~min}$. The pellet was resuspended in half of MS concentration and the OD was adjusted to 0.8 . The bacterial suspension was used to overexpress CRMYC1 transiently using the agroinfiltration method. The mature Catharanthus leaves were infected with recombinant bacteria using a $1 \mathrm{ml}$ insulin syringe. The plants were kept in the greenhouse and the leaves were harvested 48 and 72 hours after infection for further analysis.

\section{Molecular analysis of agroinfitrated plants}

Genomic DNA was extracted from the control and infected leaves by following the CTAB method. The presence of $n p t$ I was confirmed by comparing the PCR amplification and specific primers in infected leaves and control plant leaves. Total RNA extraction (Denazist RNA extraction kit) and DNase treatment (Thermoscientific DNAse kit) were carried out for all samples to avoid RNA contamination. First strand CDNA was synthesized (Amplisense Company) for the realtime $C r M Y C 1$ gene expression analysis. The relative real-time reaction was carried out using 1 XSyber Green buffer by employing specific primer for CrMYC1 and elongation factors $\alpha$ gene (Table 2). The elongation factor $\alpha$ gene was used as 
an internal control for data normalization. The amplification reactions were performed in a lineGenek thermal cycler (Bioer, China). The data were analyzed based on the threshold cycle (CT) method and the fold expression of CrMYC1 mRNAs was calculated by the $2^{-\Delta \Delta C T}$ method (Larionov et al., 2005; Livak and Schmittgen 2001).

\section{HPLC analysis of alkaloids}

For the purpose of biochemical analysis, the samples were freeze-dried after harvesting the leaf samples and freezing in liquid nitrogen. The dry weight was measured after lyophilization. Next, $0.1 \mathrm{gr}$ of grounded tissue was used for alkaloid. Accurately weighed samples were extracted with methanol and sonicated for $10 \mathrm{~min}$. The extracts were kept at room temperature for 12 hours. Afterward, the samples were filtered by a $0.2 \mu \mathrm{m}$ filter and then injected. The HPLC analysis was performed using a YOUNGLIN Korean device equipped with the UV730D detector, $250 \mathrm{~mm} \times 4.6 \mathrm{~mm}$ Eclipse plus C18 column. The temperature of the column was maintained at $35^{\circ} \mathrm{C}$. The injection volume was $20 \mu \mathrm{l}$ and carried out manually. The mobile phase consisted of a mixture of methanol as Solvent A, acetonitrile as Solvent B, ammonium acetate with $25 \mathrm{mM}$ concentration, and $0.1 \%$ trimethylamine as solvent C (15:45:40). The HPLC analysis was carried out at $1 \mathrm{~mL} / \mathrm{min}$ flow rate (Ferreres et al., 2010; Siddiqui et al., 2011; Zhang et al., 2014).

The standard markers vincristine, vinblastine, ajmalicine, and catharanthine were purchased from Sigma (Sigma, USA). The samples were analyzed two times with two biological repeats for each (totally 4 times) and the results were presented as the mean \pm standard deviation (SD).

\section{Statistical analysis}

All data in this research were statistically analyzed using the LSD test and the least difference between treatments was determined with a confidence interval of $95 \%$. For real-time analysis, in addition to doing the experiments in a negative control and five standard samples, they were repeated at least 3 times under identical conditions. For HPLC analysis, the samples were analyzed two times with two biological repeats for each (totally 4 times) and the results were presented as the mean \pm standard deviation (SD).

\section{Conclusion}

According to the findings of this research, CrMYC1 is a suitable candidate for TIA enrichment in the metabolic engineering program of $C$. roseus. The overexpression of this gene causes an increase in the yield of the most important terpenoid indole alkaloid including catharanthine, vinblastine, and vincristine. Moreover, based on the results obtained from agroinfiltration method for transient gene expression in Catharanthus, this method is introduced as a reliable and short-term consuming system for expression of TIA genes as a primary step in gene transfer and manipulation of $C$. roseus.

\section{Acknowledgement}

The authors are grateful to the Biotechnology Institute of Shiraz University and Central Lab of Agriculture and Natural
Resources College (University of Tehran) for technical support of this research. The authors declare no conflict of interest.

\section{References}

Broun P, Liu Y, Queen E, Schwarz Y, Abenes ML, Leibman M (2006) Importance of transcription factors in the regulation of plant secondary metabolism and their relevance to the control of terpenoid accumulation. Phytochem Rev. 5:27-38.

Chatel G, Montiel G, Pre M, Memelink J, Thiersault M, Saint-Pierre B, Doireau P, Gantet P (2003) CrMYC1, a Catharanthus roseus elicitor-and jasmonate-responsive bHLH transcription factor that binds the G-box element of the strictosidine synthase gene promoter. J Exp Bot. 54:2587-2588.

Di Fiore S, Hoppmann V, Fischer R, Schillberg S (2004) Transient gene expression of recombinant terpenoid indole alkaloid enzymes in Catharanthus roseus leaves. Plant Mol Biol Report. 22:15-22.

Dutta A, Singh D, Kumar S, Sen J (2007) Transcript profiling of terpenoid indole alkaloid pathway genes and regulators reveals strong expression of repressors in Catharanthus roseus cell cultures. Plant Cell Rep. 26:907-915.

El-Sayed M, Verpoorte R (2007) Catharanthus terpenoid indole alkaloids: biosynthesis and regulation. Phytochem Rev. 6:277-305.

Ferreres F, Pereira DM, Valentão $P$, Oliveira JM, Faria J, Gaspar L, Sottomayor M, Andrade PB (2010) Simple and reproducible HPLC-DAD-ESI-MS/MS analysis of alkaloids in Catharanthus roseus roots. J Pharm Biomed Anal. 51:6569.

Gidding C, Kellie S, Kamps W, De Graaf S (1999) Vincristine revisited. Agric Biol Chem. 51:1311-1317.

Hirata K, Yamanaka A, Kurano N, Miyamoto K, Miura Y (1987) Production of indol alkaloids in multiple shoot culture of Catharanthus roseus (L). G. Don. Agric Biol Chem. 51:1311-1317.

Jacobs DI, Snoeijer W, Hallard D, Verpoorte R (2004) The Catharanthus alkaloids: pharmacognosy and biotechnology. Curr Med Chem. 11:607-628.

Larionov A, Krause A, Miller W (2005) A standard curve based method for relative real time PCR data processing. BMC bioinformatics. 6:62-66.

Li CY, Leopold AL, Sander GW, Shanks JV, Zhao L, Gibson SI (2013) The ORCA2 transcription factor plays a key role in regulation of the terpenoid indole alkaloid pathway. BMC Plant Biol. 13:155-172.

Livak KJ, Schmittgen TD (2001) Analysis of relative gene expression data using real-time quantitative PCR and the 2- $\triangle \Delta C T$ method. methods. 25:402-408.

Memelink J, Verpoorte R, Kijne JW (2001) ORCAnization of jasmonate-responsive gene expression in alkaloid metabolism. Trends Plant Sci. 6:212-219.

Menke FL, Champion A, Kijne JW, Memelink J (1999) A novel jasmonate-and elicitor-responsive element in the periwinkle secondary metabolite biosynthetic gene Str interacts with a jasmonate-and elicitor-inducible AP2-domain transcription factor, ORCA2. EMBO J. 18:4455-4463.

Mujib A, Ilah A, Aslam J, Fatima S, Siddiqui ZH, Maqsood M (2012) Catharanthus roseus alkaloids: application of 
biotechnology for improving yield. Plant Growth Regul. 68:111-127.

Nammi S, Boini MK, Lodagala SD, Behara RBS (2003) The juice of fresh leaves of Catharanthus roseus Linn. reduces blood glucose in normal and alloxan diabetic rabbits. BMC Complement Altern Med. 3:1-4.

Pauwels L, Inze D, Goossens A (2009) Jasmonate-inducible gene: what does it mean. Trends Plant Sci. 14:87-91.

Pre M, Siberil Y, Memelink J, Champion A, Doireau P, Gantet $P$ (2000) Isolation by the yeast one-hybrid system of cDNAs encoding transcription factors that bind to the Gbox element of the strictosidine synthase gene promoter from Catharanthus roseus. Int J Bio-Chromatogr. 5:229244.

Shanks JV (2005) Phytochemical engineering: combining chemical reaction engineering with plant science. AIChE journal. 51:2-7.

Siddiqui MJA, Ismail Z, Saidan NH (2011) Simultaneous determination of secondary metabolites from Vinca rosea plant extractives by reverse phase high performance liquid chromatography. Pharmacogn Mag. 7:92-96.

Suttipanta N, Pattanaik S, Kulshrestha M, Patra B, Singh SK, Yuan L (2011) The transcription factor CrWRKY1 positively regulates the terpenoid indole alkaloid biosynthesis in Catharanthus roseus. Plant Physiol. 157:2081-2093.

Tiong SH, Looi CY, Hazni H, Arya A, Paydar M, Wong WF, Cheah S-C, Mustafa MR, Awang K (2013) Antidiabetic and antioxidant properties of alkaloids from Catharanthus roseus (L.) G. Don. Molecules. 18:9770-9784.

Van der Fits L, Zhang H, Menke FL, Deneka M, Memelink J (2000) A Catharanthus roseus BPF-1 homologue interacts with an elicitor-responsive region of the secondary metabolite biosynthetic gene Str and is induced by elicitor via a JA-independent signal transduction pathway. Plant Mol Biol. 44:675-685.

Verma M, Ghangal R, Sharma R, Sinha AK, Jain M (2014) Transcriptome analysis of Catharanthus roseus for gene discovery and expression profiling. PloS one. 9:e103583.

Zhang H, Hedhili S, Montiel G, Zhang Y, Chatel G, Pré M, Gantet P, Memelink J (2011) The basic helix-loop-helix transcription factor CrMYC2 controls the jasmonate-responsive expression of the ORCA genes that regulate alkaloid biosynthesis in Catharanthus roseus. Plant J. 67:61-71.

Zhang L, Qing-Hui G, Yuan-Gang Z, Lei Y, Yu-Liang M, Yang L (2014) Simultaneous quantitative determination of five alkaloids in Catharanthus roseus by HPLC-ESI-MS/MS. Chin J Nat Med. 12:786-793.

Zhao J, Verpoorte R (2007) Manipulating indole alkaloid production by Catharanthus roseus cell cultures in bioreactors: from biochemical processing to metabolic engineering. Phytochem Rev. 6:435-457.

Zhao L, Sander GW, Shanks JV (2013) Perspectives of the metabolic engineering of terpenoid indole alkaloids in Catharanthus roseus hairy roots. Adv Biochem Eng Biotechnol. 134:23-54.

Zhou ML, Shao JR, Tang YX (2009) Production and metabolic engineering of terpenoid indole alkaloids in cell cultures of the medicinal plant Catharanthus roseus (L.) G. Don (Madagascar periwinkle). Applied Biochem. 52:313-323.

Zhu X, Zeng X, Sun C, Chen S (2014) Biosynthetic pathway of terpenoid indole alkaloids in Catharanthus roseus. Front Med.8:285-293. 